Review Article

\title{
Toward the Era of a One-Stop Imaging Service Using an Angiography Suite for Neurovascular Disorders
}

\author{
Sheng-Che Hung, ${ }^{1,2}$ Chung-Jung Lin, ${ }^{1,2}$ Wan-Yuo Guo, ${ }^{1,2}$ Feng-Chi Chang, \\ Chao-Bao Luo, ${ }^{1,2}$ Michael Mu-Huo Teng, ${ }^{1,2}$ and Cheng-Yen Chang ${ }^{1,2}$ \\ ${ }^{1}$ Department of Radiology, Taipei Veterans General Hospital, 201, Section 2, Shih-Pai Road, Taipei 112, Taiwan \\ ${ }^{2}$ National Yang-Ming University, Taipei 112, Taiwan
}

Correspondence should be addressed to Wan-Yuo Guo; wyguo@vghtpe.gov.tw

Received 28 January 2013; Revised 21 April 2013; Accepted 23 April 2013

Academic Editor: Fan-Lin Kong

Copyright (C) 2013 Sheng-Che Hung et al. This is an open access article distributed under the Creative Commons Attribution License, which permits unrestricted use, distribution, and reproduction in any medium, provided the original work is properly cited.

\begin{abstract}
Transportation of patients requiring multiple diagnostic and imaging-guided therapeutic modalities is unavoidable in current radiological practice. This clinical scenario causes time delays and increased risk in the management of stroke and other neurovascular emergencies. Since the emergence of flat-detector technology in imaging practice in recent decades, studies have proven that flat-detector X-ray angiography in conjunction with contrast medium injection and specialized reconstruction algorithms can provide not only high-quality and high-resolution CT-like images but also functional information. This improvement in imaging technology allows quantitative assessment of intracranial hemodynamics and, subsequently in the same imaging session, provides treatment guidance for patients with neurovascular disorders by using only a flat-detector angiographic suite- a so-called one-stop quantitative imaging service (OSIS). In this paper, we review the recent developments in the field of flatdetector imaging and share our experience of applying this technology in neurovascular disorders such as acute ischemic stroke, cerebral aneurysm, and stenoocclusive carotid diseases.
\end{abstract}

\section{An Actual Clinical Scenario of Stroke Management}

A 67-year-old man presented at the hospital emergency unit with symptoms of acute right hemiplegia of less than 6 hours' duration. After a rapid assessment, the patient was taken to the computed tomography (CT) room, where noncontrast CT scan excluded intracranial hemorrhage. Immediate CT angiography depicted an occlusion at the proximal portion of the left middle cerebral artery, and a subsequent perfusion study identified a large penumbra, which manifested as prolonged time to peak (TTP) and preserved cerebral blood flow $(\mathrm{CBF})$.

Because of the proximal cerebral artery occlusion and the risk of cell death in a large area of brain parenchyma, the patient was sent to the angiographic suite for revascularization with intra-arterial approach. After an interventional procedure lasting more than an hour, the occluded left middle cerebral artery was opened by mechanical thrombectomy and a few smaller occluded branches were left untreated. The patient was then sent back to the stroke intensive care unit. He was followed up on the next day by CT angiography and perfusion imaging using multidetector CT (MDCT). The studies showed normal hemodynamic parameters in most of the penumbra and no hemorrhage in the brain parenchyma. He was discharged a few days later under a favorable clinical status with only minor neurological deficits.

\section{Introduction}

Recently, angiographic suites equipped with flat detectors have become a standard imaging practice. Flat-detector imaging is also known as flat-detector computed tomography (FDCT) or angiographic CT. Remarkable advances in imaging technology over the recent years have resulted in 
notable improvement of imaging acquisition and postprocessing techniques in FDCT. In addition to digital subtraction angiography (DSA), which is obtained by subtraction of images before from after contrast medium injection and removing superimposed bone and soft tissue densities [1], FDCT can provide CT-like brain parenchyma images (Dyn$\mathrm{aCT}$ ) and three-dimensional morphological and hemodynamic datasets of vasculatures, by combining one or more $\mathrm{C}$-arm rotations. Consequently, a one-stop peritherapeutic imaging service has become feasible.

In this paper, we review the recent progress of FDCT technology in angiographic suite and share our clinical experience in coupling these imaging techniques with the clinical workflow of neurovascular disorders.

\section{Technical Principles of Flat-Detector CT}

A state-of-the-art flat panel detector consisted of two independent layers. The first is a fluorescence scintillator screen of cesium iodide, which absorbs and converts the X-rays into light photons, and the second is a layer of photodiodes, made up of hydrogenated amorphous silicon to convert light photons into a digital signal $[2,3]$. The original development of flat-panel detectors was aimed at improving standard radiography by providing a higher dynamic range and a fast and repeated direct digital readout. With the improvement of three-dimensional reconstruction techniques [4-7] and reduction of artifacts, the concept of applying flat detectors for X-ray computed tomography had been investigated for several years. The FDCT can be installed in a gantry similar to that of a conventional MDCT [8], or on a C-arm system. The $\mathrm{C}$-arm based system is named because of its configuration and used primarily for fluoroscopic imaging during surgical and angiographic procedures. It can be immobile, mounted on the floor or ceiling of the angiographic room, or mobile that can operate in any medical scenario, for example, operation theater or intensive care unit. In this paper, the term FDCT refers to an angiographic suite equipped with a C-arm system and a flat detector. The system can generate CT images by a series of projection data over an angular range of 180 degrees plus fan angle. If equipped with a large size detector to cover a wide field of view, for example, 40 by $40 \mathrm{~cm}^{2}$, the FDCT can obtain a large scanning volume in a single rotation. Thus, the term of cone beam FDCT is interchangeably used in the literature [9].

The earlier works of C-arm CT where originally performed by using an conventional image intensifier system in 1990s [10-12]. However, because of inherent limitations, namely, low dynamic range, image distortion, and lowcontrast detectability, the application was limited to $3 \mathrm{D}$ rotational angiography that allowed visualizing high-contrast vessels typically by employing intra-arterial contrast medium injections $[12,13]$. Compared with image intensifiers, flat detectors offer superior image quality, including improved detective quantum efficiency (DQE), modulation transfer function (MTF), dynamic range, and dose efficiency [14]. Furthermore, a C-arm system equipped with flat detectors is capable of providing projection radiography, fluoroscopy, DSA, and CT-like images in one imaging suite. Thus, the Carm FDCT gains wide popularity in current imaging practice because of the versatile applications in the angiographic suite $[15,16]$.

\section{Angiographic Suite Equipped with FDCT}

An angiographic suite equipped with FDCT enables early recognition of intracranial complications during endovascular therapeutic procedures, such as coiling an intracranial aneurysm, stenting a stenotic artery, or other interventional procedures $[17,18]$. With its high spatial imaging resolution and capacity for correcting metallic artifacts, DynaCT can detect most brain parenchymal hemorrhages in emergency situations $[18,19]$. The combination of DSA with DynaCT has proven superior to two-dimensional or even threedimensional DSA alone for the management of neuroendovascular complications [20].

In conjunction with intra-arterial or intravenous contrast medium injection, FDCT angiography provides images with higher spatial resolution than MDCT angiography does. Moreover, FDCT is able to directly demonstrate the relationship between endovascular devices or vascular malformations and the surrounding parenchymal structures for guiding a treatment or planning a therapeutic strategy. The direct demonstration has not been possible since the invention of conventional DSA early in the last century $[21,22]$.

\section{Functional Imaging}

For functional analysis of hemodynamics, the contrast medium injection protocol and imaging reconstruction algorithm of FDCT are modified and optimized. By subtracting a mask rotation run from a contrast medium-filled rotation run, in which contrast medium in tissues reaches a static state after bolus contrast medium injection, FDCT enables measurement of parenchymal cerebral blood volume (FDCTPBV) $[23,24]$. In a preliminary study by Struffert et al., the CBV values obtained with FDCT-PBV were strongly correlated to those obtained with MDCT. The mean difference of CBV values between FDCT and MDCT was small $(0.04 \pm 0.55 \mathrm{~mL} / 100 \mathrm{~mL})$ [25]. On-site CBV measurement enables peritherapeutic monitoring of hemodynamics and allows a timely management when endovascular treatments are encountered.

Advancements in protocols of contrast medium injection, imaging acquisition, rotational speed, and reconstruction algorithms have improved remarkably the temporal resolution of C-arm FDCT for hemodynamic measurement. A recent study by Ganguly et al. demonstrated the feasibility of measuring CBF, CBV, and mean transit time (MTT) directly with C-arm FDCT. The hemodynamic maps generated from $\mathrm{C}$-arm FDCT correlated well with CT perfusion maps from MDCT [26]. 


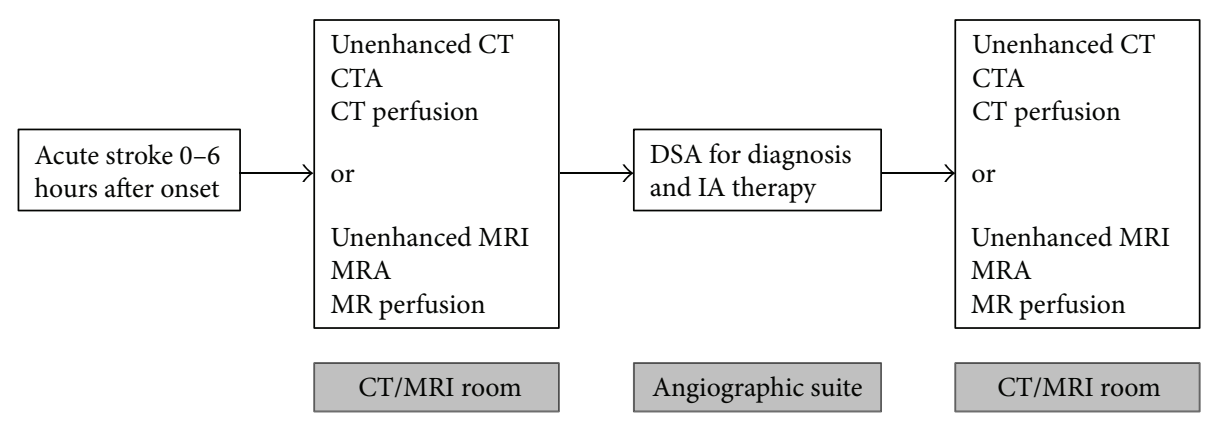

(a) Typical stroke management protocol

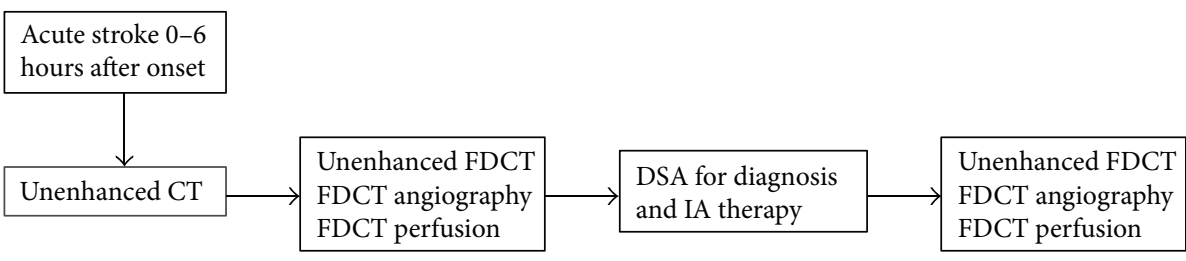

\section{CT room Angiographic suite}

(b) Potential one-stop shop stroke management protocol in the angiographic suite

FIGURE 1: Shift of clinical paradigm in stroke management by employing flat-detector angiographic suite.

\section{Clinical Experience}

6.1. Acute Ischemic Stroke. According to the World Health Organization (WHO), stroke is the second leading cause of death worldwide (10.8\%). Furthermore, human neural tissues are rapidly and irreversibly lost at an estimated rate of 1.9 million neurons each minute as stroke progresses [27]. These facts emphasize the importance of emergent management of stroke both from medical and public health aspects although patient's clinical status at presentation and appropriate treatments being given (medical, endovascular, and surgical approaches) determine the final clinical outcome.

Clinically, the time interval between ictus and hospital arrival determines the treatment option in stroke management. Advancements in imaging technology and imaging expertise, however, play a role in modifying the treatment paradigm. Magnetic resonance imaging and CT provide morphological and functional data regarding brain tissues that correlate well with stroke in the temporal and spatial domains. This information forms the foundation for treatment options. The evolving clinical scenario toward earlier diagnosis, prompter revascularization of occluded vessels, more timely tissue salvage, and better neurological function preservation becomes clinically appealing. Consequently, FDCT, as a one-stop imaging technique, provides pretherapeutic anatomic and physiological information for diagnosis and minimizes the time interval between diagnosis and revascularization procedure when a subsequent interventional neurovascular procedure is dynamically needed.
By combining C-arm rotational acquisition with intraarterial contrast medium injection from the aortic arch, we can obtain (1) noncontrast DynaCT, (2) a three-dimensional volume of intracranial vasculature, and (3) an FDCT-PBV map. Noncontrast DynaCT helps detect intracranial hemorrhage at any peritherapeutic time point. DynaCT reliably detected intracerebral hematomas with an overall sensitivity up to $93.3 \%$ in a study of 44 patients [19], but lower when hematomas were small, located in the posterior fossa or adjacent to the skull base. DynaCT is also less sensitive to detect perimesencephalic subarachnoid hemorrhages (SAH) or minimal intraventricular hemorrhage. Peritherapeutic FDCT-PBV maps help identify the infarct core immediately before mechanical thrombectomy, guide the treatment decision, and predict final infarct size immediately following revascularization $[28,29]$ (Figure 1).

A partly hypothetical clinical scenario of stroke management of the same patient as reported in the first paragraph is as follows.

A 67-year-old man arrives at the hospital emergency unit with symptoms of acute right hemiplegia of less than 6 hours' duration. After clinical evaluation and exclusion of intracranial hemorrhage by initial noncontrast MDCT, the patient is directly transferred to an FDCT angiographic suite for vascular and perfusion imaging, where preparation for the revascularization procedure starts simultaneously. FDCT angiography shows total occlusion of the left middle cerebral artery and FDCT-PBV demonstrates an area of hypoperfusion in the left frontoparietal lobes. An intra-arterial revascularization procedure by mechanical thrombectomy 


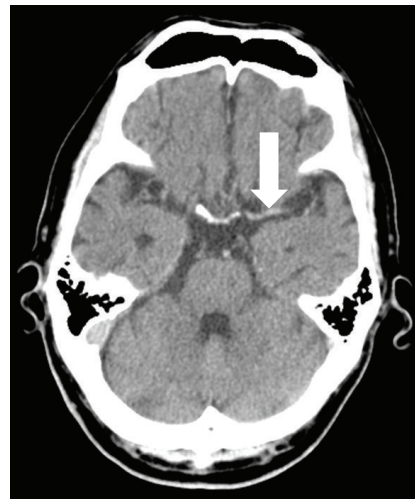

(a)

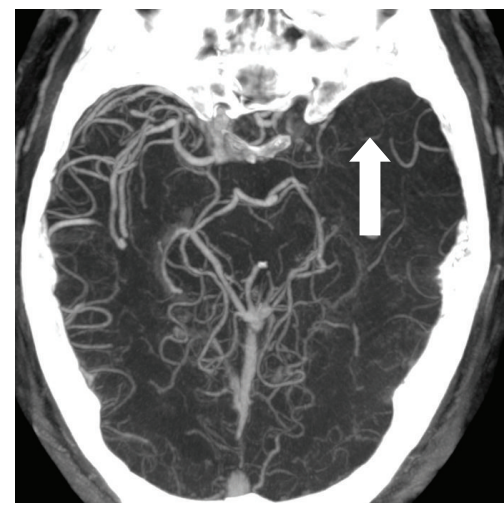

(b)

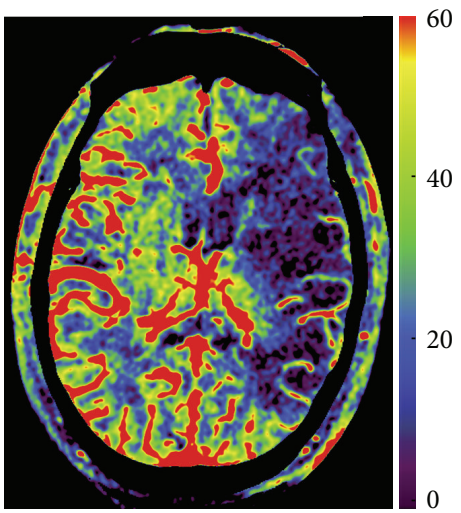

(c)

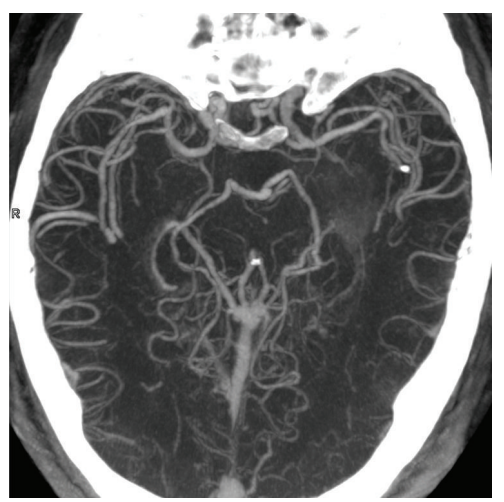

(d)

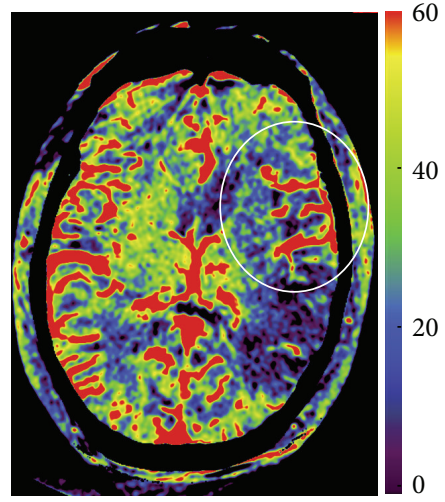

(e)

Figure 2: A 67-year-old man with left middle cerebral artery (MCA) occlusion. (a) Noncontrast computed tomography (CT) demonstrated a hyperdense MCA sign (arrow) and excluded intracranial hemorrhage. (b) Flat-detector CT (FDCT) angiography demonstrated the total occlusion of left MCA (arrow). (c) A parenchymal cerebral blood volume (FDCT-PBV) map depicted a large area of hypoperfusion in the corresponding left MCA territory, which was similar to the results of multidetector CT perfusion imaging (not shown). (d) After intra-arterial mechanical thrombectomy, recanalization of the left MCA was demonstrated by FDCT angiography. (e) An FDCT-PBV map depicted the recovery of $\mathrm{CBV}$ values (circle) in part of the hypoperfused parenchyma after revascularization.

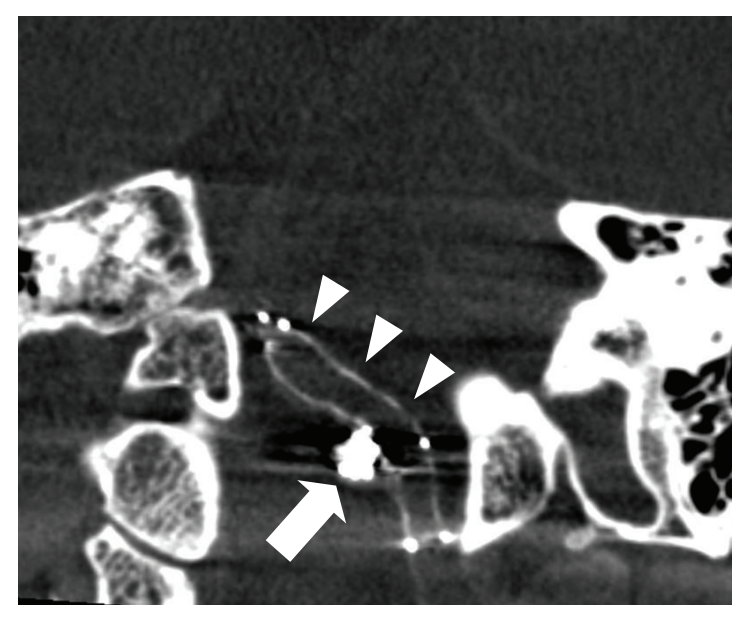

Figure 3: Intraprocedural DynaCT showed the relationship between stent struts (arrowheads) and coil mass (arrow) of a 50year-old woman who received stent-assisted embolization for a left posterior inferior cerebellar artery aneurysm. starts immediately. The occluded middle cerebral artery is opened in less than an hour. A few smaller occluded branches are left untreated. Immediately after revascularization, noncontrast DynaCT excludes intracranial hemorrhage. After the endovascular treatment, the patient is sent back to the stroke intensive care unit. Clinically, the posttherapeutic course is smooth. No imaging followup is requested. The patient is discharged a few days later with only minor neurological deficits and favorable clinical status (Figure 2).

6.2. Cerebral Aneurysm. Since the International Subarachnoid Aneurysm Trial (ISAT) and the Analysis of Treatment by Endovascular Approach of Nonruptured Aneurysms (ATENA), endovascular treatment has been established as a first-line treatment in the management of ruptured and nonruptured aneurysms $[30,31]$.

In our practice, DynaCT can be used to evaluate the degree of hydrocephalus and visualize the position of a shunt [32], monitor the extent of SAH immediately before and after 


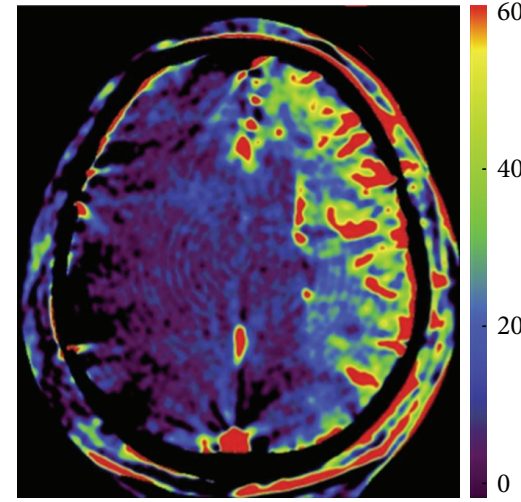

(a)

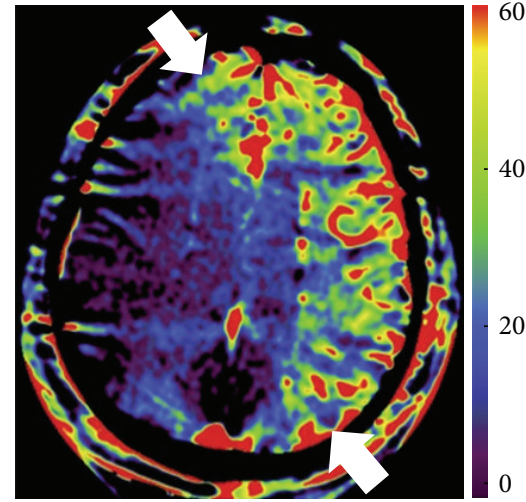

(b)

Figure 4: A 77-year-old man who underwent carotid artery stenting for left internal carotid artery high-grade stenosis. Prestenting (a) and poststenting (b) parenchymal cerebral blood volume (FDCT-PBV) maps in conjunction with selective intra-arterial contrast medium injection demonstrated increased CBV values and arterial territorial shifting (arrows).

endovascular procedures, and exclude intracranial complications before transferring the patient back to the ward from an angiographic suite [33]. For patients with broad-based cerebral aneurysms requiring stent-assisted coil embolization or flow diverter stents, precise confirmation of their positioning and relationship is important for endovascular treatment. However, stent struts are not radiopaque, and only the proximal and distal radiopaque stent markers are visible in fluoroscopy. Fluoroscopic localization and detection of stent morphology are difficult after deployment. Intraprocedural DynaCT enhances visualization of stent positioning with high spatial and contrast resolution and clearly illustrates the relationship between stent struts and coil mass (Figure 3). In a cohort study of eleven patients undergoing stent-assisted aneurysm embolization, the stent visibility was excellent in small aneurysms. However, the visibility was deteriorated in aneurysms larger than $10 \mathrm{~mm}$ in diameter due to beam hardening artifacts [34]. Besides, DynaCT enables early recognition of procedure-related complications, such as incomplete stent deployment, stent migration, stent fracture, and coil dislocation [35-37].

One of the future applications of the one-stop angiography suite is management of cerebral vasospasm. Cerebral vasospasm with delayed cerebral ischemia is the leading cause of morbidity and mortality in patients with aneurysmal SAH and survived from the initial hemorrhage. DSA is used as the reference standard, but not all angiographic vasospasms are clinically symptomatic. A combination of CT angiography and CT perfusion is reported to yield high diagnostic accuracy and can potentially improve the diagnosis of cerebral vasospasm after $\mathrm{SAH}$ [38]. Our preliminary experience shows that FDCT-PBV maps may help to identify severely ischemic brain parenchyma. By reformatting the sources images of FDCT-PBV, we can detect mild or moderate stenosis in proximal cerebral arteries with sensitivities of $84.7 \%$ and $90 \%$ by two independent raters, respectively, in a series of consecutive ten exams. This may optimize the treatment protocol of chemical angioplasty, which involves intra-arterial infusion of nimodipine, a dihydropyridine calcium channel blocker.
6.3. Stenoocclusive Carotid Disease. In symptomatic intracranial atherosclerotic disease, intracranial angioplasty and stenting are increasingly used as a therapeutic option [3942]. Similar to the situation in stent-assisted coil embolization of intracranial aneurysms, DynaCT and FDCT angiography are capable of visualizing the strut of a stent per se and the surroundings during the procedure [37, 43]. Moreover, the peritherapeutic FDCT-PBV maps demonstrate hemodynamic improvements in CBV values and arterial territory shifting (Figure 4 ). We found that peritherapeutic CBV changes were inconsistent and variable, which concurred with previous studies $[44,45]$.

With the high incidence of in-stent restenosis (8\%-30\%) [46-48], regular imaging followup is mandatory for patients who have undergone intracranial stenting for their stenoocclusive arteries. DSA, although invasive, is the standard follow-up imaging. FDCT angiography with intravenous contrast medium injection is less invasive than transarterial DSA and provides superior spatial resolution compared with MDCT angiography in both parenchymal and vascular imaging [49].

\section{Conclusion}

In conclusion, FDCT angiographic suite provides one-stop imaging for neurovascular disorders with the following advantages.

7.1. On-Site Assessment of Peritherapeutic Intracranial Conditions. Intracranial conditions, for example, ventricular size and hemorrhage, may occur or change after emergent treatments or deteriorate between/during the procedures of endovascular treatment. Moreover, the hemodynamic status and infarct core may dynamically evolve along the time course of stroke. The freedom of reassessing brain morphology and hemodynamics at any time during the endovascular procedure of stroke treatment provides a precise and updated intracranial roadmap for timely tailoring of treatment plan. 
7.2. Prompt Initiation of Intra-Arterial Revascularization Treatment. Selected patients, for example, those with suspected acute ischemic stroke ( $<6$ hours) or suspected postSAH vasospasm, could be brought directly to an FDCT angiographic suite where DynaCT, FDCT angiography, and FDCT-PBV could be obtained with one stop. The imaging service algorithm could optimize the overall workflow of stroke management by avoiding patient relocation among imaging scanners and data transferal. The new paradigm might minimize the risk and time delay of interventional procedures.

\section{Acknowledgments}

This research is cosponsored by Taipei Veterans General Hospital and Siemens Healthcare (Grant no. T1100200) and in part supported by NSC Grant 101-2314-B-075-047-MY2 and Taipei Veterans General Hospital Grant V102C-115.

\section{References}

[1] D. P. Harrington, L. M. Boxt, and P. D. Murray, "Digital subtraction angiography: overview of technical principles," American Journal of Roentgenology, vol. 139, no. 4, pp. 781-786, 1982.

[2] M. Kamran, S. Nagaraja, and J. V. Byrne, "C-arm flat detector computed tomography: the technique and its applications in interventional neuro-radiology," Neuroradiology, vol. 52, no. 4, pp. 319-327, 2010.

[3] U. Neitzel, "Status and prospects of digital detector technology for CR and DR," Radiation Protection Dosimetry, vol. 114, no. 1-3, pp. 32-38, 2005.

[4] R. Fahrig and D. W. Holdsworth, "Three-dimensional computed tomographic reconstruction using a C-arm mounted XRII: image-based correction of gantry motion nonidealities," Medical Physics, vol. 27, no. 1, pp. 30-38, 2000.

[5] M. J. Daly, J. H. Siewerdsen, Y. B. Cho, D. A. Jaffray, and J. C. Irish, "Geometric calibration of a mobile C-arm for intraoperative cone-beam CT," Medical Physics, vol. 35, no. 5, pp. 2124-2136, 2008.

[6] Y. Cho, D. J. Moseley, J. H. Siewerdsen, and D. A. Jaffray, "Accurate technique for complete geometric calibration of conebeam computed tomography systems," Medical Physics, vol. 32, no. 4, pp. 968-983, 2005.

[7] F. Noo, R. Clackdoyle, C. Mennessier, T. A. White, and T. J. Roney, "Analytic method based on identification of ellipse parameters for scanner calibration in cone-beam tomography," Physics in Medicine and Biology, vol. 45, no. 11, pp. 3489-3508, 2000.

[8] R. Gupta, A. C. Cheung, S. H. Bartling et al., "Flat-panel volume CT: fundamental principles, technology, and applications," Radiographics, vol. 28, no. 7, pp. 2009-2022, 2008.

[9] J. H. Siewerdsen, "Cone-beam CT with a flat-panel detector: fsrom image science to image-guided surgery," Nuclear Instruments and Methods in Physics Research A, vol. 648, no. 1, pp. S241-S250, 2011.

[10] R. Fahrig, A. J. Fox, S. Lownie, and D. W. Holdsworth, "Use of a C-arm system to generate true three-dimensional computed rotational angiograms: preliminary in vitro and in vivo results," American Journal of Neuroradiology, vol. 18, no. 8, pp. 1507-1514, 1997.
[11] U. Linsenmaier, C. Rock, E. Euler et al., “Three-dimensional CT with a modified C-arm image intensifier: feasibility," Radiology, vol. 224, no. 1, pp. 286-292, 2002.

[12] M. Grass, R. Koppe, E. Klotz et al., “Three-dimensional reconstruction of high contrast objects using C-arm image intensifier projection data," Computerized Medical Imaging and Graphics, vol. 23, no. 6, pp. 311-321, 1999.

[13] W. A. Kalender and Y. Kyriakou, "Flat-detector computed tomography (FD-CT)," European Radiology, vol. 17, no. 11, pp. 2767-2779, 2007.

[14] A. R. Cowen, A. G. Davies, and M. U. Sivananthan, “The design and imaging characteristics of dynamic, solid-state, flat-panel $\mathrm{x}$-ray image detectors for digital fluoroscopy and fluorography," Clinical Radiology, vol. 63, no. 10, pp. 1073-1085, 2008.

[15] D. A. Jaffray and J. H. Siewerdsen, "Cone-beam computed tomography with a flat-panel imager: initial performance characterization," Medical Physics, vol. 27, no. 6, pp. 1311-1323, 2000.

[16] Y. Kyriakou, T. Struffert, A. Dörfler, and W. A. Kalender, "Basics priciples of flat detector computed tomography (FDCT)," Radiologe, vol. 49, no. 9, pp. 811-819, 2009.

[17] H. J. Cloft and D. F. Kallmes, "Cerebral aneurysm perforations complicating therapy with Guglielmi detachable coils: a metaanalysis," American Journal of Neuroradiology, vol. 23, no. 10, pp. 1706-1709, 2002.

[18] A. Dörfler, T. Struffert, T. Engelhorn, and C. Richter, "Rotational flat-panel computed tomography in diagnostic and interventional neuroradiology," RoFo, vol. 180, no. 10, pp. 891-898, 2008.

[19] T. Struffert, G. Richter, T. Engelhorn et al., "Visualisation of intracerebral haemorrhage with flat-detector CT compared to multislice CT: results in 44 cases," European Radiology, vol. 19, no. 3, pp. 619-625, 2009.

[20] N. S. Heran, J. K. Song, K. Namba, W. Smith, Y. Niimi, and A. Berenstein, "The utility of DynaCT in neuroendovascular procedures," American Journal of Neuroradiology, vol. 27, no. 2, pp. 330-332, 2006.

[21] T. Struffert, S. Kloska, T. Engelhorn et al., "Optimized intravenous Flat Detector CT for non-invasive visualization of intracranial stents: first results," European Radiology, vol. 21, no. 2, pp. 411-418, 2011.

[22] M. R. Levitt, D. L. Cooke, B. V. Ghodke, L. J. Kim, D. K. Hallam, and L. N. Sekhar, "Stent view' flat-detector CT and stent-assisted treatment strategies for complex intracranial aneurysms," World Neurosurgery, vol. 75, no. 2, pp. 275-278, 2011.

[23] M. Zellerhoff, Y. Deuerling-Zheng, C. M. Strother et al., "Measurement of cerebral blood volume using angiographic Carm systems," in Medical Imaging 2009: Biomedical Applications in Molecular, Structural, and Functional Imaging, vol. 7262 of Proceedings of SPIE, pp. 72620H-1-72620H-8, 2009.

[24] T. Struffert, Y. Deuerling-Zheng, S. Kloska et al., "Cerebral blood volume imaging by flat detector computed tomography in comparison to conventional multislice perfusion CT," European Radiology, vol. 21, no. 4, pp. 882-889, 2011.

[25] T. Struffert, Y. Deuerling-Zheng, S. Kloska et al., "Flat detector CT in the evaluation of brain parenchyma, intracranial vasculature, and cerebral blood volume: a pilot study in patients with acute symptoms of cerebral ischemia," American Journal of Neuroradiology, vol. 31, no. 8, pp. 1462-1469, 2010.

[26] A. Ganguly, A. Fieselmann, M. Marks et al., "Cerebral CT perfusion using an interventional C-arm imaging system: cerebral blood flow measurements," American Journal of Neuroradiology, vol. 32, no. 8, pp. 1525-1531, 2011. 
[27] J. L. Saver, “Time is brain-quantified," Stroke, vol. 37, no. 1, pp. 263-266, 2006.

[28] P. Mordasini, M. El-Koussy, C. Brekenfeld et al., "Applicability of tableside flat panel detector CT parenchymal cerebral blood volume measurement in neurovascular interventions: preliminary clinical experience," American Journal of Neuroradiology, vol. 33, no. 1, pp. 154-158, 2012.

[29] C. J. Lin, M. Yu, S. C. Hung et al., "In-room assessment of cerebral blood volume for guidance during intra-arterial thrombolytic therapy," Interventional Neuroradiology, vol. 18, no. 4, pp. 463-468, 2012.

[30] A. Molyneux, R. Kerr, I. Stratton et al., "International Subarachnoid Aneurysm Trial (ISAT) of neurosurgical clipping versus endovascular coiling in 2143 patients with ruptured intracranial aneurysms: a randomized trial," Journal of Stroke and Cerebrovascular Diseases, vol. 11, no. 6, pp. 304-314, 2002.

[31] L. Pierot, L. Spelle, and F. Vitry, "ATENA: the first prospective, multicentric evaluation of the endovascular treatment of unruptured intracranial aneurysms," Journal of Neuroradiology, vol. 35, no. 2, pp. 67-70, 2008.

[32] P. von Gottberg, M. Psychogios, G. Schuetze et al., "Feasibility of flat panel detector computed tomography for position assessment of external ventricular drainage," Neurologia i Neurochirurgia Polska, vol. 47, no. 1, pp. 32-42, 2013.

[33] M. Doelken, T. Struffert, G. Richter et al., "Flat-panel detector volumetric CT for visualization of subarachnoid hemorrhage and ventricles: preliminary results compared to conventional CT," Neuroradiology, vol. 50, no. 6, pp. 517-523, 2008.

[34] G. Richter, T. Engelhorn, T. Struffert et al., "Flat panel detector angiographic CT for stent-assisted coil embolization of broadbased cerebral aneurysms," American Journal of Neuroradiology, vol. 28, no. 10, pp. 1902-1908, 2007.

[35] J. H. Buhk, K. Kallenberg, A. Mohr, P. Dechent, and M. Knauth, "Evaluation of angiographic computed tomography in the follow-up after endovascular treatment of cerebral aneurysms-a comparative study with DSA and TOF-MRA," European Radiology, vol. 19, no. 2, pp. 430-436, 2009.

[36] F. Clarencon, M. Piotin, S. Pistocchi, D. Babic, and R. Blanc, "Evaluation of stent visibility by flat panel detector CT in patients treated for intracranial aneurysms," Neuroradiology, vol. 54, no. 10, pp. 1121-1125, 2012.

[37] P. Mordasini, F. Al-Senani, J. Gralla, D. D. Do, C. Brekenfeld, and G. Schroth, "The use of flat panel angioCT (DynaCT) for navigation through a deformed and fractured carotid stent," Neuroradiology, vol. 52, no. 7, pp. 629-632, 2010.

[38] E. D. Greenberg, R. Gold, M. Reichman et al., "Diagnostic accuracy of CT angiography and CT perfusion for cerebral vasospasm: a meta-analysis," American Journal of Neuroradiology, vol. 31, no. 10, pp. 1853-1860, 2010.

[39] L. Zhang, Q. Huang, Y. Zhang et al., "A single-center study of Wingspan stents for symptomatic atherosclerotic stenosis of the middle cerebral artery," Journal of Clinical Neuroscience, vol. 20, no. 3, pp. 362-366, 2013.

[40] Y. S. Shin, B. M. Kim, S. H. Suh et al., "Wingspan stenting for intracranial atherosclerotic stenosis: clinical outcomes and risk factors for in-stent restenosis," Neurosurgery, vol. 72, no. 4, pp. 596-604, 2013.

[41] Y. Zhou, Q. W. Yang, and H. Y. Xiong, "Angioplasty with stenting for intracranial atherosclerosis: a systematic review," Journal of International Medical Research, vol. 40, no. 1, pp. 18-27, 2012.
[42] D. J. Fiorella, A. S. Turk, E. I. Levy et al., "US wingspan registry: 12-month follow-up results," Stroke, vol. 42, no. 7, pp. 1976-1981, 2011.

[43] M. N. Psychogios, P. Schramm, J. H. Buhk et al., "Angiographic CT after intravenous contrast agent application: a noninvasive follow-up tool after intracranial angioplasty and stenting," American Journal of Neuroradiology, vol. 31, no. 10, pp. 18861891, 2010.

[44] A. Waaijer, M. S. Van Leeuwen, M. J. P. Van Osch et al., "Changes in cerebral perfusion after revascularization of symptomatic carotid artery stenosis: CT measurement," Radiology, vol. 245, no. 2, pp. 541-548, 2007.

[45] Y. Duan, G. Li, Y. Yang et al., "Changes in cerebral hemodynamics after carotid stenting of symptomatic carotid artery," European Journal of Radiology, vol. 81, no. 4, pp. 744-748, 2012.

[46] H. L. Lutsep, "Stenting of Symptomatic Atherosclerotic Lesions in the Vertebral or Intracranial Arteries (SSYLVIA): study results," Stroke, vol. 35, no. 6, pp. 1388-1392, 2004.

[47] J. C. Wojak, D. C. Dunlap, K. R. Hargrave, L. A. DeAlvare, H. S. Culbertson, and J. J. Connors, "Intracranial angioplasty and stenting: long-term results from a single center," American Journal of Neuroradiology, vol. 27, no. 9, pp. 1882-1892, 2006.

[48] A. Bose, M. Hartmann, H. Henkes et al., "A novel, selfexpanding, nitinol stent in medically refractory intracranial atherosclerotic stenoses: the Wingspan study," Stroke, vol. 38, no. 5, pp. 1531-1537, 2007.

[49] J. H. Buhk, P. Lingor, and M. Knauth, "Angiographic CT with intravenous administration of contrast medium is a noninvasive option for follow-up after intracranial stenting," Neuroradiology, vol. 50, no. 4, pp. 349-354, 2008. 


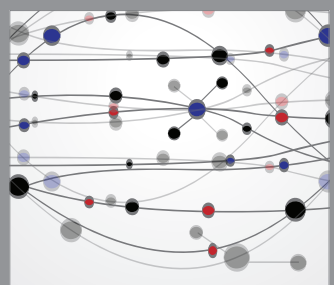

The Scientific World Journal
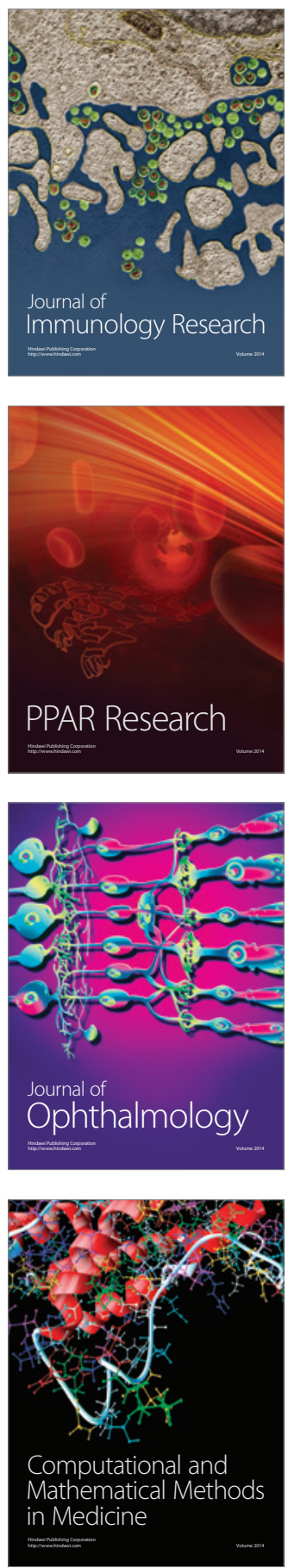

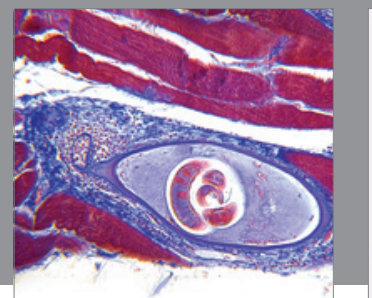

Gastroenterology

Research and Practice
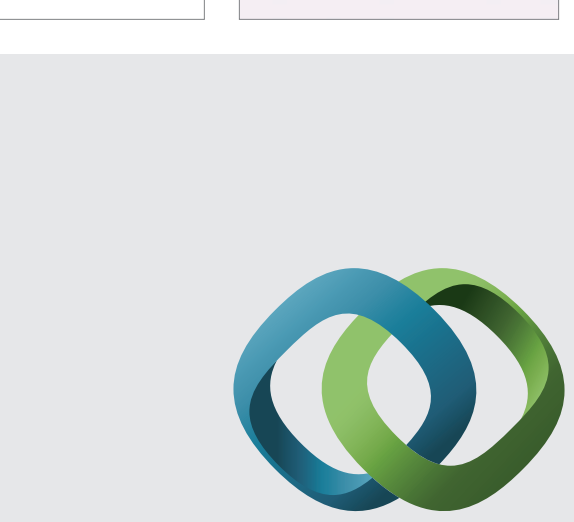

\section{Hindawi}

Submit your manuscripts at

http://www.hindawi.com
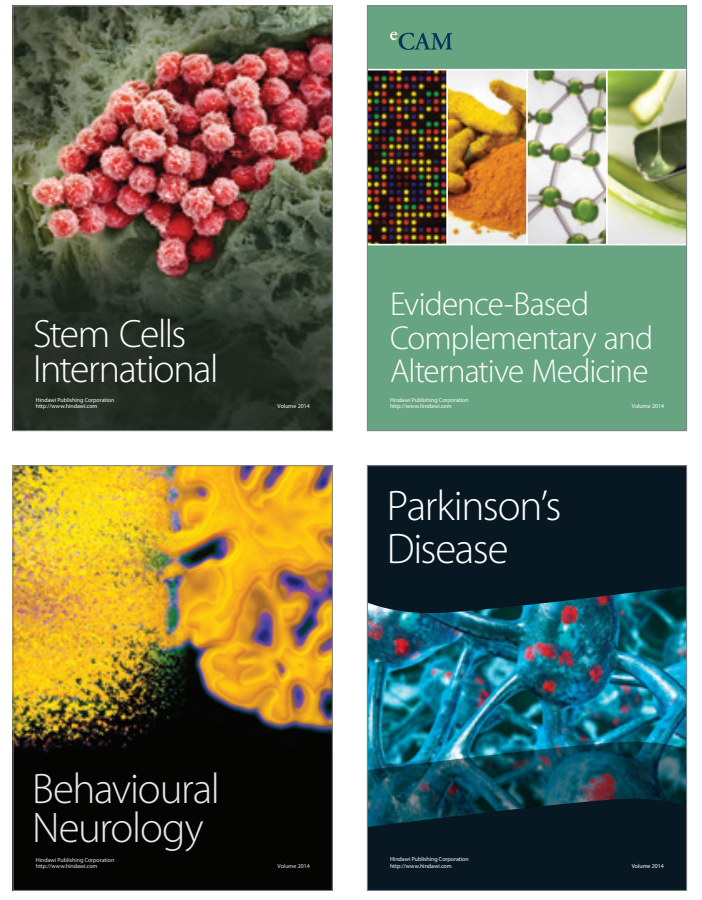
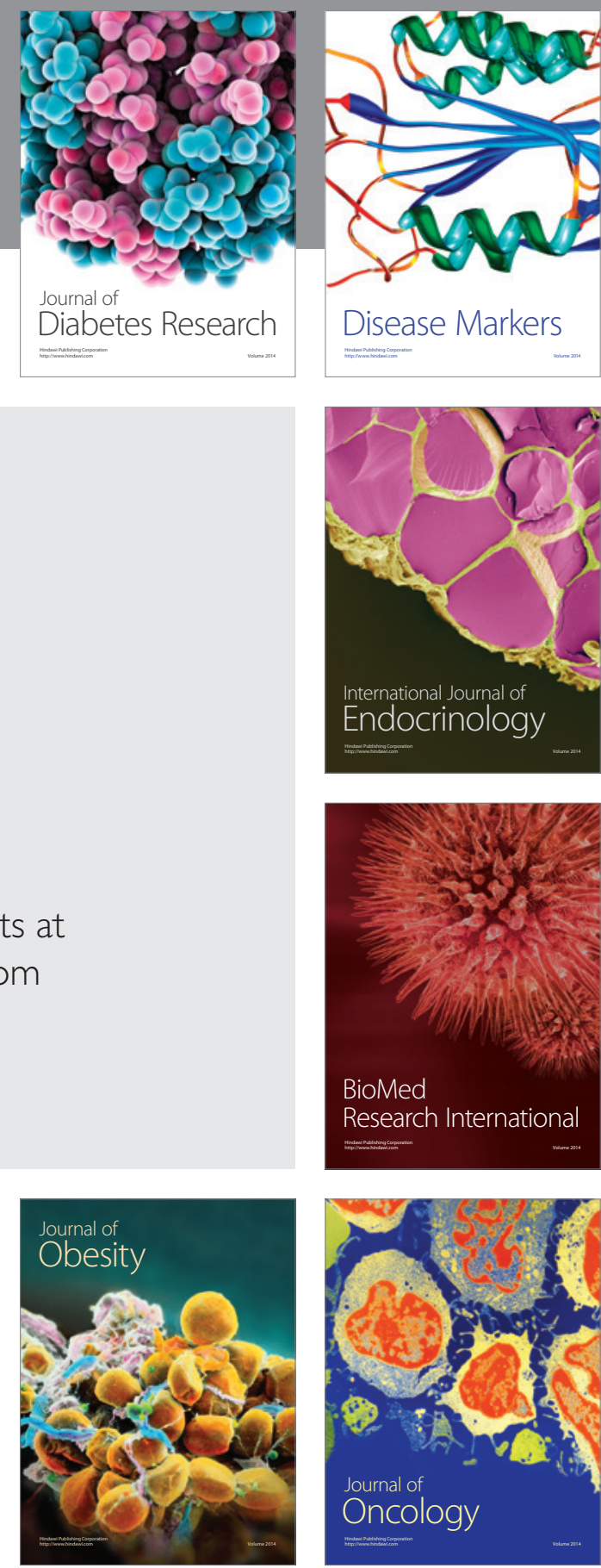

Disease Markers
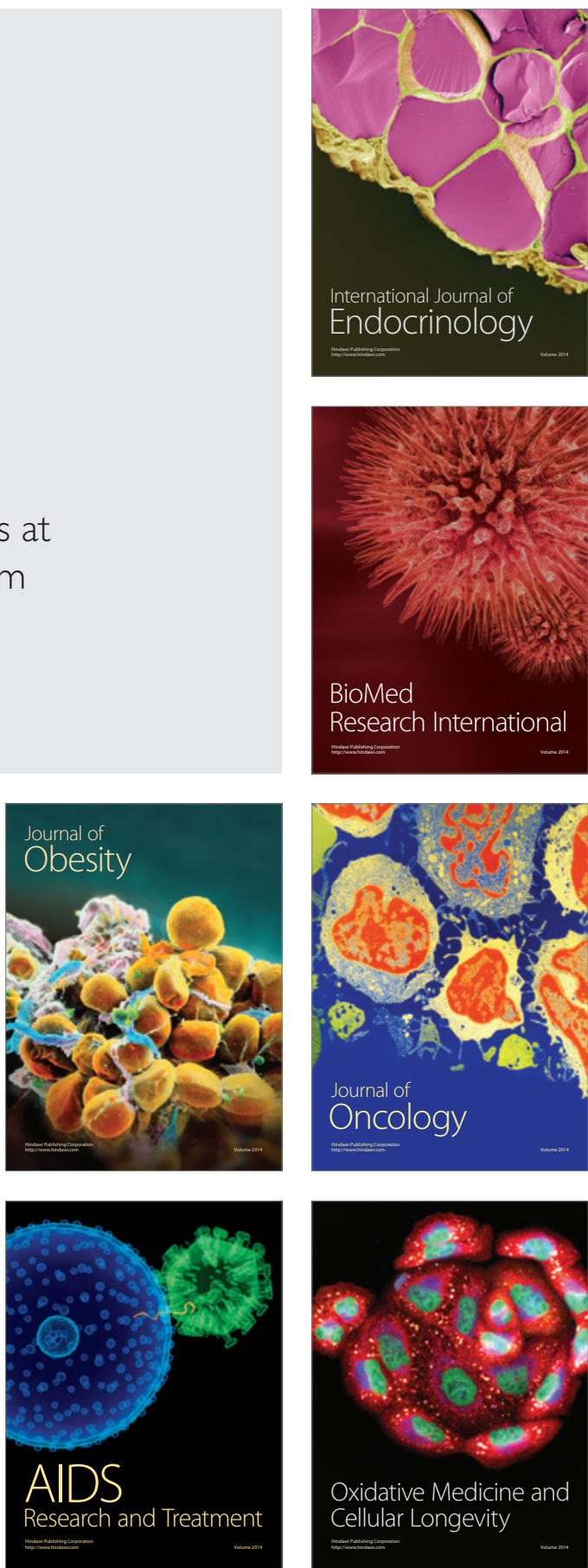